EPJ Web of Conferences 37, 06007 (2012)

DOI: $10.1051 /$ epjconf/20123706007

(C) Owned by the authors, published by EDP Sciences, 2012

\title{
Strangeness production in antiproton-nucleus annihilation
}

\author{
A.B. Larionov ${ }^{1,2,3, a}$, T. Gaitanos ${ }^{1}$, H. Lenske ${ }^{1}$, and U. Mosel ${ }^{1}$ \\ 1 Institut für Theoretische Physik, Universität Giessen, D-35392 Giessen, Germany \\ 2 National Research Center "Kurchatov Institute", 123182 Moscow, Russia \\ 3 Frankfurt Institute for Advanced Studies, J.W. Goethe-Universität, D-60438 Frankfurt am Main, \\ Germany
}

\begin{abstract}
The results of the microscopic transport calculations of $\bar{p}$-nucleus interactions within a GiBUU model are presented. The dominating mechanism of hyperon production is the strangeness exchange processes $\bar{K} N \rightarrow Y \pi$ and $\bar{K} N \rightarrow \Xi K$. The calculated rapidity spectra of $\Xi$ hyperons are significantly shifted to forward rapidities with respect to the spectra of $S=-1$ hyperons. We argue that this shift should be a sensitive test for the possible exotic mechanisms of $\bar{p}$-nucleus annihilation. The production of the double $\Lambda$-hypernuclei by $\Xi^{-}$interaction with a secondary target is calculated.
\end{abstract}

\section{Introduction}

The interest to strangeness production in $\bar{p}$-nucleus interactions was originally related to the mechanism of strangeness enhancement in a quark-gluon plasma (QGP) proposed by Rafelski and Müller in early 80's for relativistic heavy-ion collisions [1]. This idea has driven several experiments at BNL [2,3], LEAR [4] and KEK [5]. Although the following-up theoretical analyses within the intranuclear cascade (INC) models [6,7] seem to support the usual mechanism of strangeness production in terms of binary hadron-hadron collisions, the collected experimental data constitute a very useful base for testing newly developing theoretical models needed in view of forthcoming experiments with antiproton beams at FAIR. In this talk we discuss our recent results of the microscopic transport calculations of the $K_{S}^{0}, \Lambda$ - and $\Xi^{-}$-hyperon and double- $\Lambda$ hypernuclei production based on the Giessen BoltzmannUehling-Uhlenbeck (GiBUU) transport model. Sec. 2 contains some model details. Sec. 3 collects the results of our calculations. We summarize in sec. 4 .

\section{Model}

The GiBUU model [8] is a unified transport-theoretical approach capable to describe photon-, electron-, neutrino-, hadron- and nucleus-induced reactions on nuclei. To formulate transport equations, we are using here a relativistic mean-field model. The transport equations for the di fferent baryon $\dot{8}=$ $N, N^{*}, \Delta, Y, \Xi, \ldots$, respective antibaryons and mesons $\pi, \eta, \rho, \omega, K, \bar{K}, \ldots$ can be written as follows:

$$
\left(p_{0}^{*}\right)^{-1}\left[p^{* \mu} \partial_{\mu}^{x}+\left(p_{v}^{*} F_{i}^{\mu \nu}+m_{i}^{*} \partial_{x}^{\mu} m_{i}^{*}\right) \partial_{\mu}^{p^{*}}\right] f_{i}\left(x, \mathbf{p}^{*}\right)=I_{i}[\{f\}] .
$$

Here, $f_{i}\left(x, \mathbf{p}^{*}\right)$ is the phase-space distribution (or Wigner) function, $p^{*}=p-V_{i}$ is the kinetic fourmomentum, $F_{i}^{\mu \nu} \equiv \partial^{\mu} V_{i}^{\nu}-\partial^{\nu} V_{i}^{\mu}$ is the field tensor, and $m_{i}^{*}=m_{i}+S_{i}$ is the effective mass. The baryonic mean field is characterized by the scalar, $S_{i}$, and vector, $V_{i}^{\mu}$, potentials. The collision term in the r.h.s. of Eq.(1) describes the residual two-body interactions beyond mean field and resonance

\footnotetext{
a e-mail: Alexei.Larionov@theo.physik.uni-giessen.de
}

This is an Open Access article distributed under the terms of the Creative Commons Attribution License 2.0, which permits unrestricted use, distribution, and reproduction in any medium, provided the original work is properly cited. 

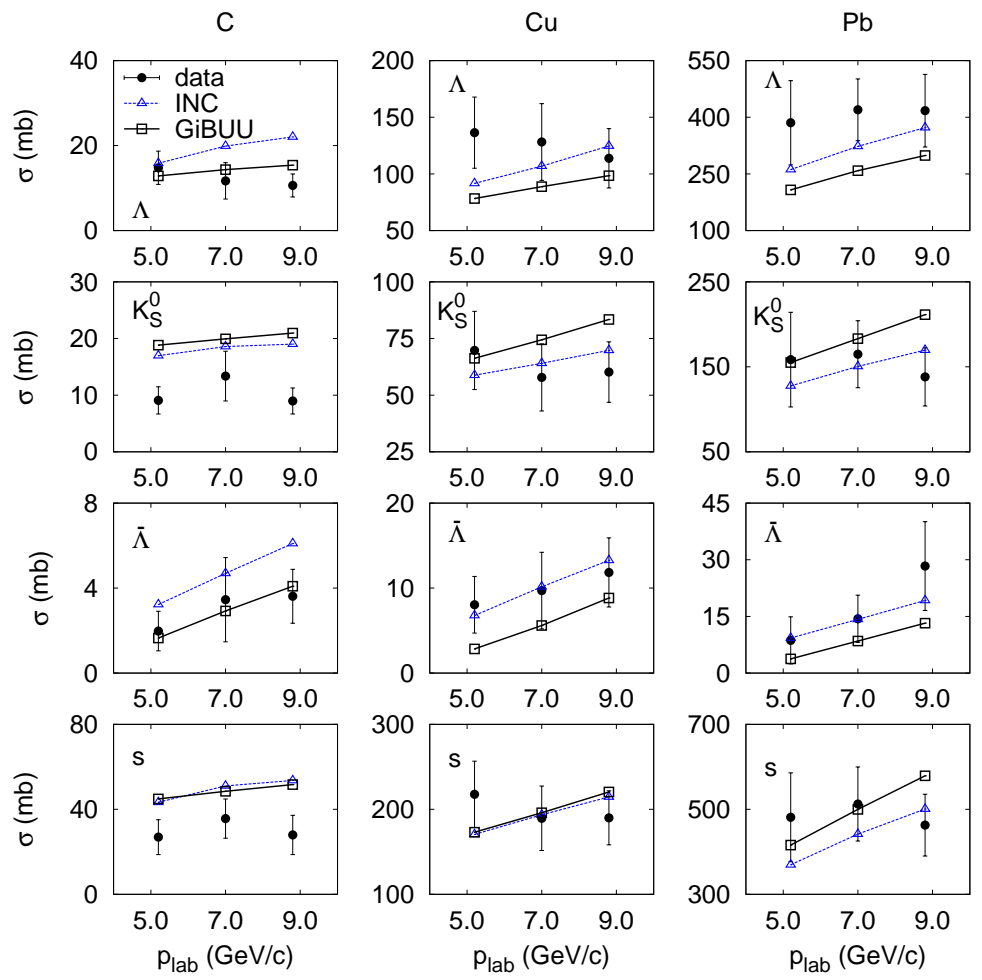

Fig. 1. Beam momentum dependence of $\Lambda, K_{S}^{0}, \bar{\Lambda}$ and strange quark production cross sections in $\bar{p}$ interactions with ${ }^{12} \mathrm{C},{ }^{64} \mathrm{Cu}$ and ${ }^{208} \mathrm{~Pb}$ nuclei. The calculated $\Lambda$ production cross sections include also the contribution of $\Lambda$ 's from $\Sigma^{0} \rightarrow \Lambda \gamma$ decays. Figure taken from [9].

decays. The two-body collision term depends on the angular differential cross sections of the particleparticle scattering and takes into account the Pauli blocking factors for the outgoing nucleons. The following relevant for the present study collision channels are included: annihilation $\bar{N} N \rightarrow$ mesons, $\bar{N} N \rightarrow \bar{N} N, \bar{N} N \rightarrow \bar{N} \Delta$ (+c.c.), $\bar{N} N \rightarrow \bar{\Lambda} \Lambda, \bar{\Sigma} \Lambda(+$ c.c), $\bar{\Xi} \Xi$. Hyperons are also produced in strangeness exchange reactions on nucleons $\bar{K} N \rightarrow Y \pi, \bar{K} N \rightarrow \Xi K$, and in the collisions of nonstrange mesons with nucleons $M N \rightarrow Y K, M=\pi, \eta, \rho, \omega$. The produced hyperons may rescatter and change their charge and/or flavour via the following processes: $\Lambda N \rightarrow \Lambda N, \Lambda N \leftrightarrow \Sigma N, \Sigma N \rightarrow \Sigma N, \Xi N \rightarrow \Xi N$, $\Xi N \rightarrow \Lambda \Lambda, \Xi N \rightarrow \Lambda \Sigma$. Further details of the model can be found in [8-10].

\section{Results}

Fig. 1 shows our results on the inclusive cross sections of neutral strange particle production in comparison with experimental data [3] and INC model calculations [7]. There is an overall satisfactory agreement of GiBUU calculations with data and with INC results. We observe, however, a systematic trend to underestimate $\Lambda$ production for heavier targets and overestimate $K_{S}^{0}$ production for light targets by transport calculations. The reason for this is not yet clear for us. One possibility is that $\bar{K}$ absorption cross sections in nuclear medium are enhanced. This suggestion is based on our observation that $\sim 60-80 \%$ of the $S=-1$ hyperon production rate is due to $\bar{K} N \rightarrow Y X, \bar{K} N \rightarrow Y^{*}$ and $\bar{K} N \rightarrow Y^{*} \pi$ reactions.

In Fig. 2 we present the rapidity spectra of $\Xi^{-}$-hyperons plotted together with the rapidity spectra of $\Lambda$-hyperons and $K_{S}^{0}$ 's. The $\Lambda$ rapidity distributions are peaked at $y \simeq 0$, because the $S=-1$ hyperons 

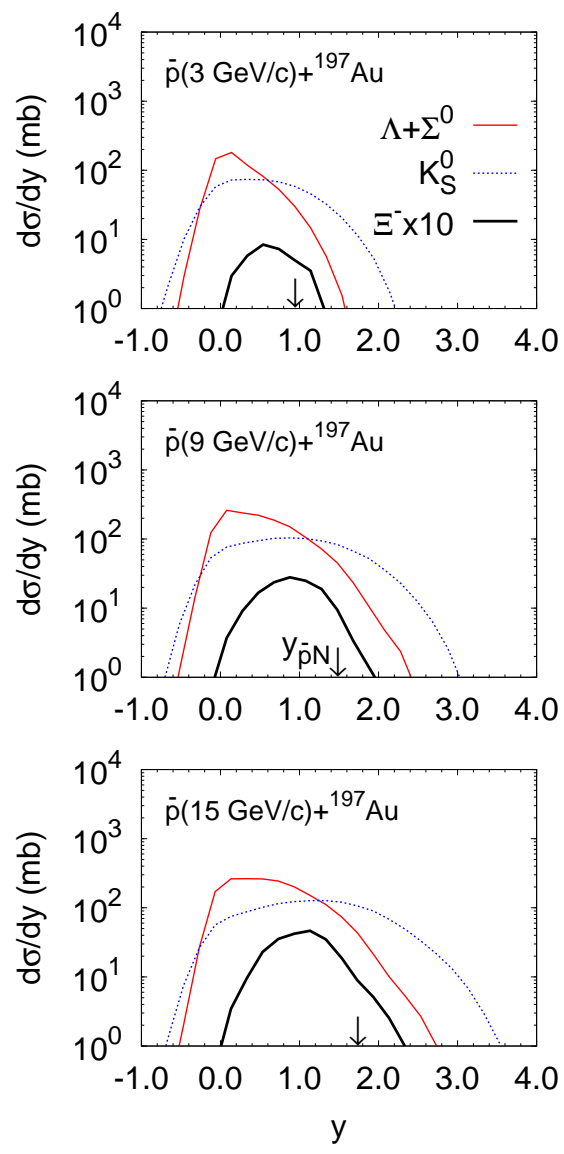

Fig. 2. Rapidity distributions of $\Xi^{-}, \Lambda$ and $K_{S}^{0}$ in $\bar{p}+{ }^{197} \mathrm{Au}$ collisions at 3,9 and $15 \mathrm{GeV} / \mathrm{c}$. The $\bar{p} N$ center-of-mass (c.m.) rapidities are marked with arrows. Figure taken from [9].

are mostly produced in the exothermic strangeness exchange reactions $\bar{K} N \rightarrow Y \pi$ with slow $\bar{K}$ 's. The rescattering on nucleons further decelerates the produced hyperons. On the other hand, the peaks of the $\Xi^{-}$rapidity distributions are shifted forwards by $0.5-1$ units of rapidity despite that the $\Xi N$ rescattering is also included in our calculations. This shift is easily explained by the dominating endothermic production channel $\bar{K} N \rightarrow \Xi K$ with the threshold $\bar{K}$ beam momentum of $1.048 \mathrm{GeV} / \mathrm{c}$ corresponding to the $\bar{K} N$ c.m. rapidity of 0.55 . We think that the difference between the peak positions of the $\Lambda$ and $\Xi$ hyperon rapidity spectra is the direct consequence of the underlying hadronic production mechanism implemented in our model and should vanish in the case of the strangeness production from the blob of a supecooled QGP [11].

The program of the future PANDA experiment [12] is indended to use a primary target to produce $\Xi^{-}$hyperons which will be then decelerated in the ordinary medium and captured to the Coulomb orbit of a secondary target nucleus. The double- $\Lambda$ hypernuclear system will be created due to reaction $\Xi^{-} p \rightarrow \Lambda \Lambda$ on a proton from the secondary target nucleus. To get some feeling of this idea, we have performed a simplified study by, first, calculating the momentum spectrum of emitted $\Xi^{-}$'s in the primary reaction and, second, by calculating the production cross section of the double- $\Lambda$ hyperfragments in the interaction of a $\Xi^{-}$with the secondary target. Fig. 3 shows the results. We see that the production cross section of double- $\Lambda$ hyperfragments grows with decreasing $\Xi^{-}$beam momentum, as expected. 


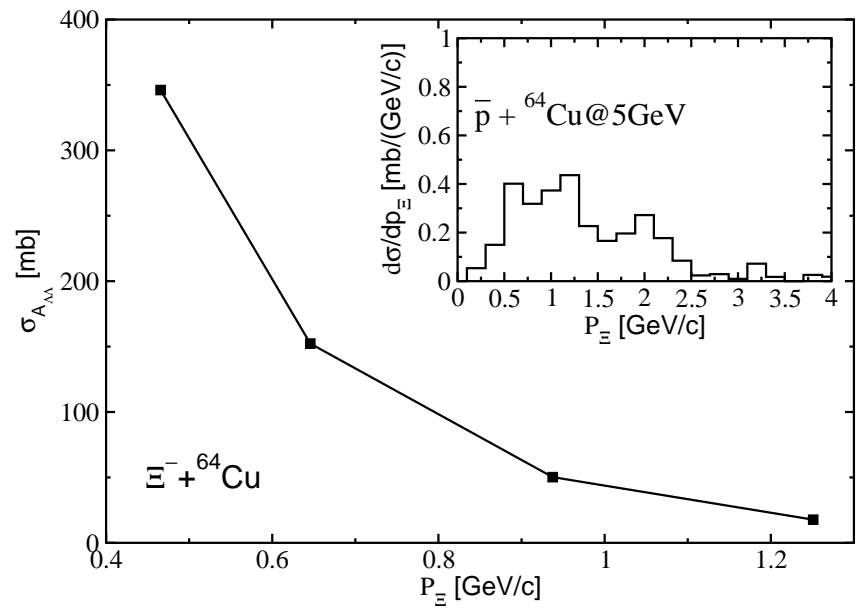

Fig. 3. Double- $\Lambda$ hyperfragment production cross section in $\Xi^{-}+{ }^{64} \mathrm{Cu}$ interactions vs beam momentum of $\Xi^{-}$. For orientation, the inset shows momentum distribution of produced $\Xi^{-}$'s in $\bar{p}+{ }^{64} \mathrm{Cu}$ interactions at $E_{\mathrm{lab}}=5 \mathrm{GeV}$. Figure taken from [10].

On the other hand, as one can see in the inset, a rather significant number of $\Xi^{-}$'s is produced at low momenta $(<1 \mathrm{GeV} / \mathrm{c})$. Alghough we have neglected the $\Xi^{-}$deceleration in the ordinary medium and its capture to the Coulomb orbit, our results support the main idea of the double- $\Lambda$ cluster production experiment.

\section{Summary}

The main conclusions of our present study are:

- Overall, GiBUU is working reasonably well for strangeness observables in $\bar{p}$-induced reactions. However, $\Lambda$ production is slightly underpredicted and $K_{S}^{0}$ production is overpredicted.

- The $S=-2$-hyperon rapidity spectra are sensitive to the underlying production mechanism: by hadronic two-body collisions or by QGP hadronization.

- Double- $\Lambda$ hyperfragment production cross section due to $\Xi^{-}$interaction with a secondary target grows with decreasing $\Xi^{-}$beam momentum reaching hundreds mb below $0.8 \mathrm{GeV} / \mathrm{c}$.

This work has been financially supported by BMBF, HIC for FAIR and DFG (Germany), and Grant NSH-7235.2010.2 (Russia).

\section{References}

1. J. Rafelski and B. Müller, Phys. Rev. Lett. 48, (1982) 1066

2. G.T. Condo et al., Phys. Rev. C 29, (1984) 1531

3. S. Ahmad et al., Nucl. Phys. Proc. Suppl. 56A, (1997) 118

4. F. Balestra et al., Phys. Lett. B 194, (1987) 192

5. K. Miyano et al., Phys. Rev. C 38, (1988) 2788

6. J. Cugnon, P. Deneye, and J. Vandermeulen, Phys. Rev. C 41, (1990) 1701

7. W.R. Gibbs and J.W. Kruk, Phys. Lett. B 237, (1990) 317

8. O. Buss et al., Phys. Rept. 512, (2012) 1; see also http://gibuu.physik.uni-giessen.de/GiBUU

9. A.B. Larionov, T. Gaitanos, and U. Mosel, Phys. Rev. C 85, (2012) 024614

10. T. Gaitanos, A.B. Larionov, H. Lenske, and U. Mosel, Nucl. Phys. A 881, (2012) 240

11. J. Rafelski, Phys. Lett. B 207, (1988) 371

12. J. Pochodzalla, Nucl. Phys. A 754, (2005) 430 